\title{
Evaluation of the Efficacy and Tolerability of a Solution Containing Propyl Betaine and Polihexanide for Wound Irrigation
}

\author{
M. Romanelli V.Dini S. Barbanera M.S. Bertone \\ Wound Healing Research Unit, Department of Dermatology, University of Pisa, Pisa, Italy
}

\section{Key Words}

Polihexanide $\cdot$ Betaine $\cdot$ Wound cleansing $\cdot$ Biofilm

\begin{abstract}
Wound cleansing represents a fundamental step in chronic wound management. Several investigations in recent years have led to a refinement of the wound cleansing protocol in order to obtain a better control of the bacterial burden during wound bed preparation and to avoid further cell and tissue damage. The aim of the present randomized controlled trial was to investigate the effects of a wound cleansing solution containing polihexanide and betaine in venous leg ulcers by means of clinical and instrumental assessment. A portable device was used on the wound bed to assess surface $\mathrm{pH}$, which has been shown to be one of the most useful non-invasive biophysical parameters in order to correlate the level of bacterial burden in different types of chronic wounds. Baseline $\mathrm{pH}$ on the wound surface (median range) was initially 8.9, and after 4 weeks of cleansing treatment and moist wound dressing was reduced and stable at 7.0 in the group treated with active cleanser. The $\mathrm{pH}$ value was significantly lower $(p<0.05)$ in this group compared to the control group at the end of the study. The treatment with the solution containing polihexanide and betaine was well tolerated by the patients and was found useful in the absorption of wound odours.

Copyright $\odot 2010$ S. Karger AG, Basel
\end{abstract}

\section{Introduction}

The importance of biofilm formation as an element of wound infection has recently been stressed [1]. When bacteria proliferate in wounds, they form microcolonies, which attach to the wound bed and secrete a glycocalyx, or any kind of extracellular matrix, and take up an interdependent surface-attached existence. These microbial communities, called 'biofilms', protect the organisms against antibiotics, antiseptics and host immune defences [2]. Almost all bacterial species form biofilm in vivo [3], thus representing a therapeutic challenge in many, if not most, bacterial diseases. Biofilm formation has also been linked to the emergence of a variety of opportunistic pathogens, such as Staphylococcus epidermidis and Pseudomonas aeruginosa, which contribute to persistent infections by changing environmental parameters [4]. Despite the recent efforts in identifying bacteria of chronic wound microflora, it is apparent that the clinical relevance resides in the structure of the bacterial community, not in the presence of particular species in the wound [5]. Experimental biofilm measurements have been developed in order to determine in vitro the ability of a bacterial strain to produce biofilm $[6,7]$. These measurements fit with the genomic presence of virulence determinants $[8,9]$. In vivo biofilm observation using a confocal laser scanning microscope is now under development [10].

In order to obtain biochemical and physical information about the wound bed and the surrounding skin, dif-

Marco Romanelli, MD, $\mathrm{PhD}$

Wound Healing Research Unit, Department of Dermatology

University of Pisa

Via Roma 67, IT-56126 Pisa (Italy)

Tel. +39 050992 436, Fax +39 050551 124, E-Mail m.romanelli@med.unipi.it 
ferent options of non-invasive and invasive measurements have been developed and tested [11]. The use of instruments to measure cutaneous parameters in wound care is in an embryonic state, and doctors and nurses still rely on the use of clinical observations. Skin lesions, and especially the way lesions evolve over time, are monitored simply according to the common sense of the caregivers, who might not use up-to-date or efficient equipment for obtaining objective measurements. Monitoring of acute and chronic wounds can be performed by measuring in an objective, precise, and reproducible way and by simply adapting the existing and proven technologies [12]. Wound measurement techniques have received consistent attention in clinical practice and research through the three main areas of interest in wound care: vascular ulcers, diabetic foot ulcers, and pressure ulcers [13]. Today, each discipline focuses on measuring the physical parameters that may contribute substantially to the development of new pathogenetic mechanisms or to the acquisition and definition of new therapeutic technologies.

The aim of our study was to evaluate the efficacy and tolerability of a solution containing propyl betaine and polihexanide in order to control the bacterial burden of chronic wounds.

\section{Materials and Methods}

This was a single-blind, monocentric, prospective, controlled, explorative comparison trial. The protocol was reviewed and accepted by the Institutional Review Board of the research facility, and all subjects read and signed an informed consent form before entering the study.

Forty patients ( 22 females and 18 males) presenting to the outpatient wound clinic of our dermatology department participated in this study. Their age range was between 55 and 73 years, with the average age being $62 \pm 3$ years. The inclusion and exclusion criteria were as follows.

Inclusion criteria:

- painful chronic leg ulcer $>8$ weeks old;

- clinical and instrumental signs of venous insufficiency;

- wound size: up to $100 \mathrm{~cm}^{2}$;

- having received compression therapy for at least 2 weeks before inclusion;

- patients over 18 years of age.

Exclusion criteria:

- allergy to one of the materials used;

- severe systemic diseases;

- acute superficial or deep vein thrombosis;

- arterial occlusive disease (stages II, III or IV);

- any arterial disease with an Ankle Brachial Pressure Index less than 0.8 ;

- immobile patient/bedridden patient;

- pregnancy and period of lactation;

- severe lymphoedema of the leg;
- diabetes with complications;

- well-known hypercoagulability;

- thrombophilia with deep vein thrombosis.

The patients were randomized with an electronic system into two groups of 20 each, and each group was assigned to the following treatment regimen and evaluated for a period of 4 weeks:

- group A: 20 patients were treated every other day with the active wound cleansing solution in association with standard wound care (polyurethane foam and elastic compression);

- group B: 20 patients were treated every other day with saline solution in association with standard wound care (polyurethane foam and elastic compression).

\section{Wound Size Measurements}

As an objective evaluation of chronic wounds, we measured the wound area with a dedicated planimetry software (Silhouette ${ }^{\circledR}$, Aranz, New Zealand). This device provides integrated imaging, measurement and documentation of the wound healing progress in a portable, non-contact system that combines a digital camera and structured lighting in the form of two laser beams to correct automatically for camera location, image scale and skin curvature, allowing rapid and accurate measurements of the wound's surface area and depth. Previous reports have quantified the repeatability of Silhouette Mobile on vascular wounds [14].

\section{Wound Surface pH Measurements}

The surface $\mathrm{pH}$ of the wound bed was measured using a flat glass electrode connected to a meter (skin pH meter HI 99181, Hanna Instruments, Italy). The instrument was applied to the wound bed with 1 or 2 drops of bidistilled water interposed between the electrode and the wound site. The electrode is applied onto the skin at intervals of $10 \mathrm{~s}$ until stabilization of the reading. Measurements were taken after dressing removal and before irrigation and cleansing of the area with the products under evaluation. Two different sites on the wound bed were evaluated and mean \pm standard deviation was taken as a final result. Measurements were also taken at a control site on normal skin of the upper chest, in an area free of hair follicles. All $\mathrm{pH}$ readings were taken at a constant room temperature of $25^{\circ} \mathrm{C}$ and relative humidity of $55 \%$. Calibration of the $\mathrm{pH}$-measuring instrument was carried out daily by means of alignment with 2 commercially available, standardized buffer solutions having a defined $\mathrm{pH}$ value (HI 70004, HI 70007, Hanna Instruments, Italy).

\section{Pain Assessment}

Patients were asked to self-assess subjectively the intensity of pain using a validated $10-\mathrm{mm}$ visual analogue scale. The pain related to the ulcer/lower limb site was defined as any kind of discomfort or painful sensation that the patient was able to describe. In order to reduce the risk of bias in the baseline assessment of pain intensity, the randomization took place after the baseline pain intensity assessment.

\section{Test Product}

Prontosan $^{\circledR}$ (B. Braun, Melsungen AG, Germany) is a wound irrigation solution, clear, colourless, containing undecylenamidopropyl betaine, polyaminopropyl biguanide, sodium hydroxide and purified water. The solution is used for cleaning wounds and for moistening and lubricating absorbent wound dressings for ulcers, burns, post-surgical wounds and abrasions. The me- 


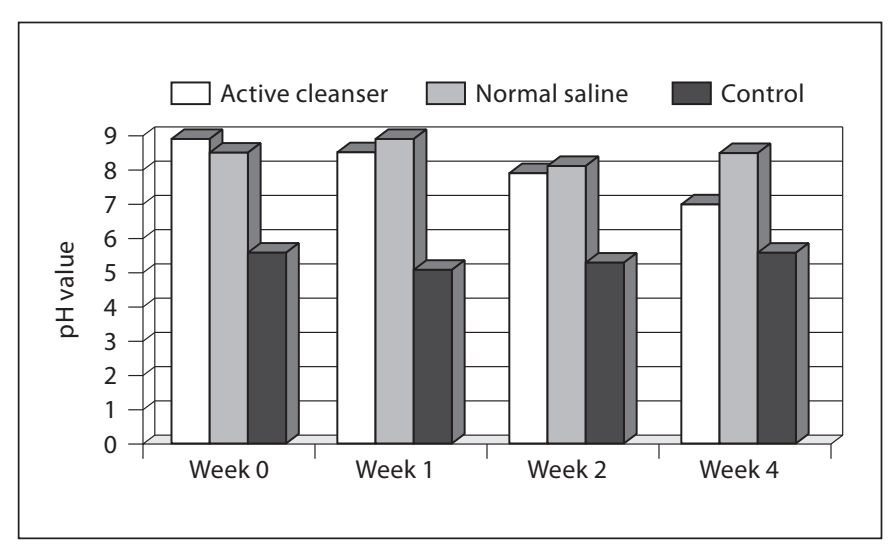

Fig. 1. Results of $\mathrm{pH}$ measurement in the wounds during the study period of 4 weeks.

chanical action of movement across the wound provides for the mechanism of action and aids in the removal of foreign material such as dirt and debris.

\section{Statistical Analysis}

The difference between the product-treated wound and the untreated wound was evaluated statistically. Analysis of variance and Student's t test were used to analyse changes in the measurements of the wound surface $\mathrm{pH}$ and pain scoring. The significance level was set at $\mathrm{p}<0.05$. The Wilcoxon test and Mann-Whitney test were used in order to analyse wound healing time and ulcer planimetry. All data were elaborated by using a commercially available statistical software package (SPSS).

\section{Results}

The treatment groups were not statistically significantly different at baseline with regard to age, mean disease duration, mean wound size or pain score. The mean duration of the disease before treatment was 24 months (range 2-192 months). Thirty-eight patients concluded the study. Two patients were lost during treatment because of change of residence; they were both from group B. At baseline, the mean $\mathrm{pH}$ value was not significantly dependent on sex or age of the patients in the two groups.

Baseline $\mathrm{pH}$ on the wound surface (median range) was initially $8.9 \pm 0.6$, and after 4 weeks of cleansing treatment and moist wound dressing was reduced and stable at $7.0 \pm 0.3$ in group $\mathrm{A}$. At the end of the study, $\mathrm{pH}$ measurement was significantly lower $(\mathrm{p}<0.05)$ in group A compared to group B (fig. 1). During the 4-week evaluation, the mean wound surface $\mathrm{pH}$ showed statistically significantly higher values $(\mathrm{p}<0.03)$ compared to normal skin.

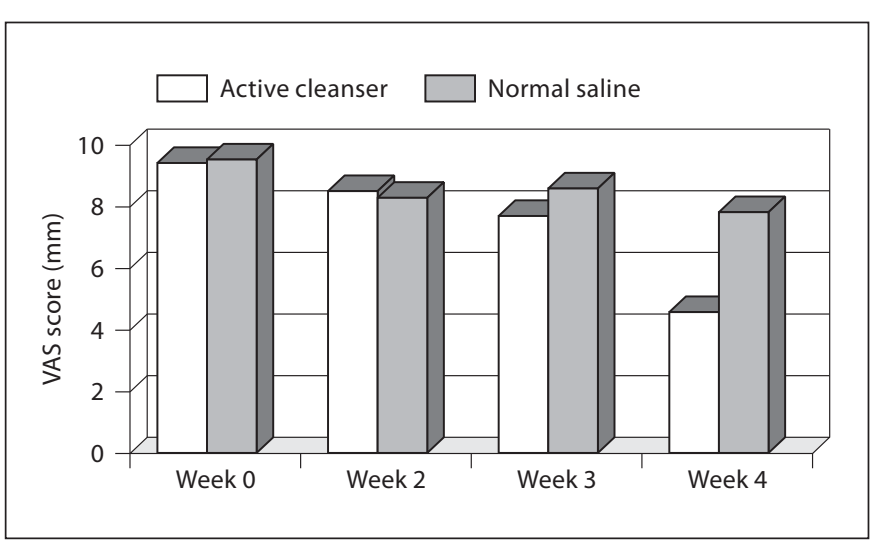

Fig. 2. Pain evaluation during treatment of venous leg ulcers before and after 4 weeks of treatment with active cleanser compared to standard therapy. VAS $=$ Visual analogue scale.

Pain control was achieved during and at the end of treatment in group A better than in group B $(p<0.05)$ (fig. 2). During treatment, patients were not affected by serious and/or unexpected adverse reactions, and there was a significant improvement of the wound bed preparation. Wound size was not statistically different in the two groups from baseline to the end of the study. We think that this was mainly due to the short period of observation. The treatment with the solution containing polihexanide and betaine was well tolerated by the patients and was found useful in the absorption of wound odours. At the end of the study, group A showed a significantly better control of the bacterial burden both clinically and by means of instrumental evaluation compared to group B.

\section{Discussion}

The role of the bacterial burden is a critical issue in wound management and is going to influence the patients' health-related quality of life. Assessment and management of the bacterial burden at dressing changes must be considered a fundamental step in wound care and must be accomplished according to the patients' expectations. It is therefore vital that practitioners are able to assess and manage bacterial burden by applying the principles of best practice available today from various consensus documents. Best local wound care will influence wound healing, and the selection of an ideal wound cleanser, according to wound status, will contribute to achieving positive outcomes and to producing a faster healing response. Modulating the wound surface $\mathrm{pH}$ to a more acidic envi- 
ronment has been shown to improve the healing rate of chronic wounds [15]. pH measurement is defined as the negative logarithm of the activity of hydrogen ions in aqueous solution, used to express acidity and alkalinity on a scale of $0-14$. The $\mathrm{pH}$ value of normal skin ranges from about 4.8 to 6.0 , depending on the presence of the so-called 'acid mantle', while the interstitial fluid shows neutral values [16]. The role of the $\mathrm{pH}$ value in wound healing has proved to be of fundamental importance, and prolonged chemical acidification of the wound bed has been shown to increase the healing rate in chronic venous leg ulcers [17]. The mechanism of interaction between an acidic $\mathrm{pH}$ and the wound healing process is related to the potential to increase tissue oxygen availability through oxygen dissociation and to reduce the histotoxicity of bacterial end products, thus stimulating the wound's healing process. $\mathrm{pH}$ values in chronic venous leg ulcers and in pressure ulcers were found to be alkaline or neutral if compared with the normal surrounding skin [18]. The change of value is in accordance with the stage of the ulcer, moving to an acidic state during the healing process [19]. Two significant methods are widely used for measuring cutaneous $\mathrm{pH}$ : the colorimetric technique and the glass electrode potentiometric measurement. The most common $\mathrm{pH}$ instrument is a flat glass electrode connected to a meter and applied to the skin, with 1 or 2 drops of bidistilled water interposed between the electrode and the skin. The use of a flat elec- trode is important in order to provide appropriate contact with the skin surface. The electrode is applied onto the skin at intervals of $10 \mathrm{~s}$ until stabilization of the reading.

The Prontosan product used in this study has already shown good efficacy and tolerability in bacterial burden control for venous ulcers with different levels of microbial involvement [20]. In this study, the quantitative assessment made by wound surface $\mathrm{pH}$ measurement was sufficiently sensitive to demonstrate an improvement of the wound surface in the Prontosan-treated wounds (fig. la, b). We think that the wound cleanser used in this study is effective on the surface of wounds, but is not able to penetrate the deeper layers of the same area. This final outcome could be of extreme value in order to speculate about biofilm control in chronic wounds, an increasing problem affecting the surface of chronic wounds, but not yet very well identified from a quantitative point of view. More studies will be necessary in order to confirm our data and to find a clinical correlation between biofilm eradication and Prontosan wound cleanser.

\section{Disclosure Statement}

This study was partially financed by B. Braun Medical AG, Switzerland. Prof. Marco Romanelli received financial support for clinical consulting from B. Braun Medical AG, Switzerland.

\section{References}

$>1$ James GA, Swogger E, Wolcott R, Pulcini ED, Secor P, Sestrich J, Costerton JW, Stewart PS: Biofilms in chronic wounds. Wound Repair Regen 2008;16:37-44.

$\checkmark 2$ Lewis K: Riddle of biofilm resistance. Antimicrob Agents Chemother 2001;45:9991007.

3 Jefferson KK: What drives bacteria to produce a biofilm? FEMS Microbiol Lett 2004; 236:163-173.

4 Costerton, JW, Stewart PS, Greenberg, EP: Bacterial biofilm: a common cause of persistent infections. Science 1999;284:1318-1322.

$\checkmark 5$ Davies CE, Hill KE, Wilson MJ, Stephens P, Hill CM, Harding KG, Thomas DW: Use of 16 ribosomal DNA PCR and denaturing gradient gel electrophoresis for analysis of the microfloras of healing and nonhealing chronic venous leg ulcers. J Clin Microbiol 2004;42:3549-3557.

6 Heydorn A, Ersboll BK, Hentzer M., Parsek MR, Givskov M, Molin S: Experimental reproducibility in flow-chamber biofilms. Microbiology 2000;146:2409-2415.

$>7$ Ando E, Monden K, Mitsuhata R, Kariyama $\mathrm{R}$, Kumon $\mathrm{H}$ : Biofilm formation among Methicillin-resistant Staphylococcus aureus isolates from patients with urinary tract infection. Acta Med Okayama 2004;58:207214.

8 Arciola CR, Baldassarri L, Montanaro L: Presence of icaA and icaD genes and slime production in a collection of staphylococcal strains from catheter-associated infections. J Clin Microbiol 2001;39:2151-2156.

$\checkmark 9$ Beenken KE, Dunman PM, McAleese F, Macapagal D, Murphy E, Projan SJ, Blevins JS, Smeltzer MS: Global gene expression in Staphylococcus aureus biofilms. J Bacteriol 2004;186:4665-4684.

10 Akiyama H. Oono T, Saito M, Iwatsuki K: Assessment of cadexomer iodine against Staphylococcus aureus biofilm in vivo and in vitro using confocal laser scanning microscopy. J Dermatol 2004;31:529-534.

11 Romanelli M, Gaggio G, Coluccia M, Riz zello F, Piaggesi A: Technological advances in wound bed measurements. Wounds 2002; 14:58-66.

12 Van Rijswijk L: Wound assessment and documentation. Wounds 1996;8:57-69.

13 Mekkes JR, Westerhof W: Image processing in the study of wound healing. Clin Dermatol 1995; 13:401-407.
14 Romanelli M, Dini V, Bertone MS, Barachini P: A new wound measurement and documentation system. J Am Acad Dermatol 2010;62:150.

15 Wilson IAI, Henry M, Quill RD, Byrne PJ: The $\mathrm{pH}$ of varicose ulcer surfaces and its relationship to healing. VASA 1979;8:339-342.

16 Dikstein S, Zlogorski A: Skin surface hydrogen ion concentration $(\mathrm{pH})$; in Leveque JL (ed): Cutaneous Investigation in Health and Disease: Noninvasive Methods and Instrumentation. York, Dekker, 1988, pp 59-78.

17 Dissemond J, Witthoff M, Brauns TC: pH values in chronic wounds. Evaluation during modern wound therapy. Hautarzt 2003;54: 959-965.

18 Glibbery AB, Mani R: pH in leg ulcers. Int J Microcirc Clin Exp 1992;2:109.

19 Tsukada K, Tokunaga K, Iwama T, Mishima Y: The $\mathrm{pH}$ changes of pressure ulcers related to the healing process of wounds. Wounds 1992;4:16-20.

20 Andriessen AE, Eberlein T: Assessment of a wound cleansing solution in the treatment of problem wounds. Wounds 2008;20:171-175. 